BMJ Paediatrics Open

\title{
Universal newborn hearing screening in South Africa: a single-centre study
}

\author{
Ayanda Gina, ${ }^{1}$ Nadja F Bednarczuk, ${ }^{2,3}$ Asitha Jayawardena, ${ }^{4}$ Peter Rea, ${ }^{5}$ \\ Qadeer Arshad (1) ,2 Yougan Saman ${ }^{1,2,5}$
}

To cite: Gina A, Bednarczuk NF, Jayawardena $A$, et al. Universal newborn hearing screening in South Africa: a single-centre study. BMJ Paediatrics Open 2021:5:e000976. doi:10.1136/ bmjpo-2020-000976

Received 7 December 2020 Accepted 16 February 2021

\section{Check for updates}

\section{(c) Author(s) (or their} employer(s)) 2021. Re-use permitted under CC BY-NC. No commercial re-use. See rights and permissions. Published by BMJ.

${ }^{1}$ School of Clinical Medicine University of KwaZulu-Natal, Durban, South Africa

${ }^{2}$ inAmind Laboratory, Department of Psychology, Neuroscience and Behaviour, University of Leicester, Leicester, UK

${ }^{3}$ King's College Hospital London Denmark Hill, SE5 9RS, London UK, London, UK

${ }^{4}$ E.N.T Department, Massachusetts Eye and Ear Infirmary, Harvard Medical School, Boston, Massachusetts, USA

${ }^{5}$ E.N.T Department, Leicester Royal Infirmary, Leicester, UK

Correspondence to DrYougan Saman; yougans@ gmail.com

\section{ABSTRACT}

Hearing screening for newborn babies is an established protocol in many high-income countries. Implementing such screening has yielded significant socioeconomic advantages at both an individual and societal level. This has yet to permeate low/middle-income countries (LMIC). Here, we illustrate how newborn hearing screening needs to be contextually adapted for effective utilisation and implementation in an LMIC. Specifically, this advocates the use of auditory brainstem testing as the first-line approach. We propose that such adaptation serves to maximise clinical efficacy and community participation at a reduced cost

\section{INTRODUCTION}

Newborn hearing screening facilitated a silent revolution for the hearing-impaired yielding significant personal, societal and economic benefits. ${ }^{1}$ However, universal screening still faces barriers, namely the need to pragmatically integrate screening with existing health infrastructure, cost considerations and access to healthcare, ${ }^{1}$ thus presenting unique challenges depending on the geographical context.

Otoacoustic emissions (OAE) and automated auditory brainstem responses (AABR) have made screening possible, with factors such as cost and training ease being the main considerations when designing a programme. ${ }^{12}$ OAEs have historically been cheap and quick but as many as $40 \%$ of babies can fail this test requiring referral to specialists for either a repeat OAE or an auditory brainstem response (ABR). ${ }^{3}$ Technological advances since the advent of screening have seen both the cost-base and time required to perform AABR considerably reduced ${ }^{4}$ making this an attractive first-line option. Such a proposition is strengthened when considering the cost of follow-up, poor compliance due to limited access to healthcare and maternal anxiety being decisive factors.

Accordingly, in settings where early discharge is the norm and access to healthcare is poor, we propose that it may be more effective to screen neonates with AABR as the first-line tool.

\section{METHODS}

Two thousand two hundred sixty-nine healthy neonates were recruited from the maternity wards following written parental consent in Amajuba district, KwaZulu-Natal, South Africa. Babies were generally tested within hours of birth as healthy babies are discharged at 6 hours post delivery. Testing was always performed in a silent room within the hospital.

To assess the ABR and OAE for each neonate, we used the Path-Sentiero-AdvancedScreener (Landsberger, Germany). For auditory brainstem testing, we implemented a chirp stimulus (broadband $1-8 \mathrm{kHz}$ ) with alternating polarity and a stimulus rate of $85 \mathrm{~Hz}$ and sound level of $35 \mathrm{dBHL}{ }^{5}$ For transient OAE, a non-linear broadband click stimulus was presented at a fixed sound level of $80 \mathrm{db}$ sound pressure level (SPL). ${ }^{6}$ All testing was performed by an audiologist with expertise in paediatric testing (AG), assisted by two research nurses.

\section{Patient and public involvement statement}

After identification of the community's needs, the researchers engaged in consultation with the department of health district and hospital managers who were in full support of potentially developing a screening programme. Patients were not involved in the design of the study as it was incorporated into the existing maternal and child healthcare services.

\section{RESULTS}

We observed that in neonates screened with ABR, 2120 babies passed the test, and 149 of the cohort failed the screening. Contrastingly, the OAE test passed only 655 babies in the cohort and failed 1614 babies. Accordingly, OAE testing would have resulted in most babies needing referral. 
DISCUSSION

Our findings highlight that adopting the OAE first protocol in our sample would have resulted in 1465 unnecessary referrals, imparting significant financial burden on both the individual and the healthcare system as well as considerable anxiety for already disenfranchised parents. Although the cost base of ABR testing is higher, when factoring in not only the equipment costs but also the costs associated with consumables and maintenance, this can effectively be mitigated against by the volume of avoidable referrals. We thus highlight the need for contextually relevant screening as a prerequisite to effectively engage all stakeholders including the families, government services and clinicians in order for such programmes to be deemed viable. Ideally, children need to be screened prior to discharge as the birth hospital is the ideal setting to ensure compliance. For a service to be successful in the South African context where early hospital discharge is the norm, screening programmes have to adjust to ensure uptake.

These findings have a wider contextual implication, as healthcare resources across the board are continually being stretched. Thus, a critical spotlight is currently being placed on expensive initiatives such as screening programmes to adapt to simultaneously maximise clinical efficacy and community participation at an everreducing cost.

Acknowledgements First, we would like to sincerely thank all the mothers for agreeing to participate in this study. Also, we would like to thank the two brilliant research nurses Zama Khubheka and Thembi Nkosi as well as Precious Buthelezi - the district rehabilitation coordinator for KZN Department of Health. Finally, they would like to thank the EU for funding this project and the Red Cross Air mercy service for assistance with travel.
Contributors All authors contributed to this manuscript in line with the set out guidelines in the Journals policy.

Funding EuropeAid/134258/M/ACT/ZA Cfp2/2014/15/87.

Competing interests None declared.

Patient and public involvement Patients and/or the public were not involved in the design, or conduct, or reporting, or dissemination plans of this research.

Patient consent for publication Not required.

Ethics approval University of KwaZulu-Natal Biomedical-Research Ethics Committee (BREC: BFC260/16(Sub-study: BFC412/15)).

Provenance and peer review Not commissioned; externally peer reviewed.

Open access This is an open access article distributed in accordance with the Creative Commons Attribution Non Commercial (CC BY-NC 4.0) license, which permits others to distribute, remix, adapt, build upon this work non-commercially, and license their derivative works on different terms, provided the original work is properly cited, appropriate credit is given, any changes made indicated, and the use is non-commercial. See: http://creativecommons.org/licenses/by-nc/4.0/.

ORCID iD

Qadeer Arshad http://orcid.org/0000-0002-6849-6685

\section{REFERENCES}

1 Morton CC, Nance WE. Newborn hearing screening - a silent revolution. N Engl J Med Overseas Ed 2006;354:2151-64.

2 The Joint Committee on Infant Hearing. Year 2019 position statement: principles and guidelines for early hearing detection and intervention programs. The Journal of Early Hearing Detection and Intervention 2019;4:1-44.

3 Heidari S, Manesh AO, Rajabi F. The sensitivity and specificity of automated auditory brainstem response and otoacoustic emission in neonatal hearing screening: a systematic review. Auditory and vestibular research 2015;24.

$4 \mathrm{Hsu} \mathrm{H-C}$, Lee F-P, Huang H-M. Results of a 1-year governmentfunded newborn hearing screening program in Taiwan. Laryngoscope 2013;123:1275-8.

5 Gina A, Bednarczuk NF, Jayawardena A, et al. Association between in utero HIV exposure and auditory brainstem response. Neurology 2020;95:928-30.

6 Meena RS, Meena D, Babu D, et al. Role of transient evoked otoacoustic emission beyond screening of hearing impairment: a study of 400 cases. Indian J Otolaryngol Head Neck Surg 2013;65:134-9. 